\title{
Tolerance, safety, and efficacy of PEG3350 + bisacodyl bowel preparation: comparison between two treatments of different duration in pediatric patients
}

\author{
Ligia Marcela Portillo Canizalez¹, Gerardo Blanco Rodriguez ${ }^{1 *}$, Gustavo Teyssier Morales ${ }^{1}$, \\ Jaime Penchyna Grub ${ }^{1}$, Sean Trauernicht Mendieta ${ }^{2}$ and Jessie Nallely Zurita-Cruz ${ }^{3}$
}

${ }^{1}$ Thoracic Surgery and Endoscopy Service, Hospital Infantil de México Federico Gómez, Mexico City, Mexico; ${ }^{2}$ Departament of Gastroenterology and Pediatric Nutrition, Hospital Infantil de México Federico Gómez, Mexico City, Mexico; ${ }^{3}$ Clinical Research Support Area, Hospital Infantil de México Federico Gómez, Mexico City, Mexico

\begin{abstract}
Background: Multiple bowel preparations have been used in children undergoing colonoscopy, with variable limitations due to acceptance, tolerance, and proper cleaning. The objective of this study was to compare the tolerability, safety, and efficacy of colonoscopy preparation with one-day of PEG 3350 (polyethylene glycol) $(4 \mathrm{~g} / \mathrm{kg} / \mathrm{day})+$ bisacodyl compared with two days of preparation with PEG 3350 (2 g/kg/day) + bisacodyl in pediatric patients. Methods: A clinical, randomized, single-blind trial was performed. Patients aged 2 to 18 years scheduled for colonoscopy were included. Patients were randomized into two groups: one day of preparation with PEG $33504 \mathrm{~g} / \mathrm{kg} / \mathrm{day}+$ bisacodyl and two days of preparation with PEG $33502 \mathrm{~g} / \mathrm{kg} / \mathrm{day}+$ bisacodyl. Through a questionnaire, a physical examination, and an endoscopic evaluation (Boston scale), the tolerance, safety and efficacy of both preparations evaluated were determined. Student's t-test was performed for quantitative variables and $\chi^{2}$ for qualitative variables. Results: There were no significant differences in compliance rates, adverse effects, and extent of colonoscopic evaluation. Conclusions: Tolerance and safety between the intestinal preparation for 1-day colonoscopy with PEG 3350 (4 g/kg/day) + bisacodyl and the 2-day preparation with PEG 3350 (2 g/kg/day) + bisacodyl were similar. The quality of cleanliness was good in both groups, being partially more effective in the 1-day group with PEG 3350 (4 g/kg/day).
\end{abstract}

Key words: Pediatric intestinal preparations. Polyethylene glycol. Bisacodyl.

Tolerancia, seguridad y eficacia de la preparación intestinal con un día de PEG3350 + bisacodilo en comparación con 2 días de PEG3350 + bisacodilo en pacientes pediátricos

\section{Resumen}

Introducción: Múltiples preparaciones intestinales se han utilizado en niños sometidos a una colonoscopia, con limitación variable debido a la aceptación, tolerancia, y la limpieza adecuada. El objetivo del estudio fue comparar la tolerancia, seguridad y eficacia de la preparación intestinal para colonoscopia de 1 día con PEG 3350 (polietilenglicol) (4 g/kg/día) +

\section{Correspondence:}

*Gerardo Blanco Rodríguez

E-mail: gerardoblancor@yahoo.com.mx
Available online: 18-06-2018 Bol Med Hosp Infant Mex. 2017;74:341-348

www.bmhim.com

2444-3409/๑ 2018. Hospital Infantil de México Federico Gómez, published by Permanyer México SA de CV, all rights reserved. 
bisacodilo en comparación con 2 días de preparación con PEG 3350 (2 g/kg/día) + bisacodilo en pacientes pediátricos. Métodos: Se realizó un ensayo clínico, aleatorizado y ciego. Se incluyeron pacientes de 2 a 18 años, que ameritaron colonoscopia en forma programada. Los pacientes se asignaron de manera aleatoria en dos grupos: 1 día de preparación con PEG $33504 \mathrm{~g} / \mathrm{kg} / \mathrm{día}$ + bisacodilo y 2 días de preparación con PEG $33502 \mathrm{~g} / \mathrm{kg} /$ día + bisacodilo. Por medio de un cuestionario, exploración física y valoración endoscópica (escala de Boston), se determinó la tolerancia, seguridad y eficacia de las 2 preparaciones a evaluar. Se realizó una prueba $T$ de student para variables cuantitativas y Chi2 para variables cualitativas. Resultados: No hubo diferencias significativas en las tasas de cumplimiento, los efectos adversos y la extensión de la evaluación colonoscópica. Conclusiones: La tolerancia y seguridad entre la preparación intestinal para colonoscopia de 1 día con PEG 3350 (polietilenglicol) (4 g/kg/día) + bisacodilo y la preparación de 2 días con PEG 3350 (2 g/kg/día) + bisacodilo fue semejante. La calidad de la limpieza fue buena en ambos grupos, siendo parcialmente más eficaz en el grupo de 1 día con PEG 3350 (polietilenglicol) (4 g/kg/día).

Palabras clave: Preparaciones intestinales pediátricas. Polietilenglicol. Bisacodilo.

\section{Introduction}

Colonoscopy in children is the adequate procedure for the diagnosis, treatment, and follow-up of disease of the large intestine and, when complemented by distal ileoscopy, is invaluable for the diagnosis and follow-up of disorders of the distal small intestine ${ }^{1}$.

An adequate colonoscopy study must fulfill certain indispensable quality requirements to ensure its maximal performance; one of them is bowel cleansing. If inadequate, there will be unexplored areas and adenomatous, polypoid lesions, but especially flat lesions, that will not be detected?

The Boston bowel preparation scale is a valid and reliable tool to evaluate cleanliness and the quality of preparation of the different segments of the colon, which was published in October 2010 in the Journal of Gastrointestinal Endoscopy. The preparation is considered to be inadequate when in any segment, the score is 0 or 1 , or when the score is lower than 6 . In these cases, colonoscopy should not be performed ${ }^{3}$ (Table 1 and Figure 1). In some reports, up to a third of patients are not adequately prepared for the exam, which leads to increased duration of the procedure, incomplete assessment, or the need to repeat the study ${ }^{3,4}$.

Agents for colonoscopy preparation can be divided in stimulating and osmotic, although some have a combined effect. Stimulating laxatives induce colonic motility through stimulation of the enteric nervous system and increase the secretion of electrolytes and water in the colon. Bisacodyl, a stimulating-type agent, is a derivative of diphenylmethane which induces contractions of great amplitude and propagation, shortening the coIonic transit time. It presents a therapeutic action by stimulating the nerve endings of the intestinal wall, inhibits absorption, and increases secretion of water and electrolytes, thus, reducing consistency and increasing fecal volume with minimal systemic absorption. In general, it is administered preferably before bed, resulting in bowel movements within 6 to 8 hours when given orally and within 30 to 60 minutes when given intrarectally. Bisacodyl is safe and effective, which is why it is used as a complement in several regimes for bowel preparation $^{5}$. Polyethylene glycol (PEG), an osmotic laxative, is a non-toxic, water-soluble polymer with minimal intestinal absorption. The commonly used compound is PEG 3350, which is a mixture of molecules of different sizes with a mean molecular weight between 3,200 and $3,700 \mathrm{~g} / \mathrm{mol}$. PEG preparations are osmotically balanced, non-absorbable and non-fermentable. These solutions induce defecation mainly by the mechanical effect of great volume lavages. The most commonly prescribed doses range from $20-40 \mathrm{ml} / \mathrm{kg}$, but no more than 1 liter/hour and until defecations are clear. It has been demonstrated that low volume preparations (2 liters or $20 \mathrm{ml} / \mathrm{kg}$ ) of PEG 3350 and bisacodyl was equally effective than full volume preparation with $4 \mathrm{li}$ ters $(40 \mathrm{ml} / \mathrm{kg})$ and was better tolerated ${ }^{6}$.

There are several inconveniences with bowelpreparation in children, which include strict dietary regimes, bad taste, large volumes of liquid and adverse effects. High doses and long preparations lead to better cleanliness but may be more bothersome to patients. Poor palatability causes nausea or vomit which may affect the patient's tolerance and, finally, the quality of cleanliness ${ }^{7}$. Every preparation for colonoscopy can be associated with adverse effects. The most common include electrolyte disorders, dehydration, nausea, vomit, pain and abdominal distention.

Multiple studies in children have reported about the harmlessness and efficacy of a 4-day bowel preparation using PEG-3350, other studies have concluded that 1 or 2 days of preparation are safe and effective. An effective dose of PEG was established based on a 
Table 1. Boston colon cleanliness scale

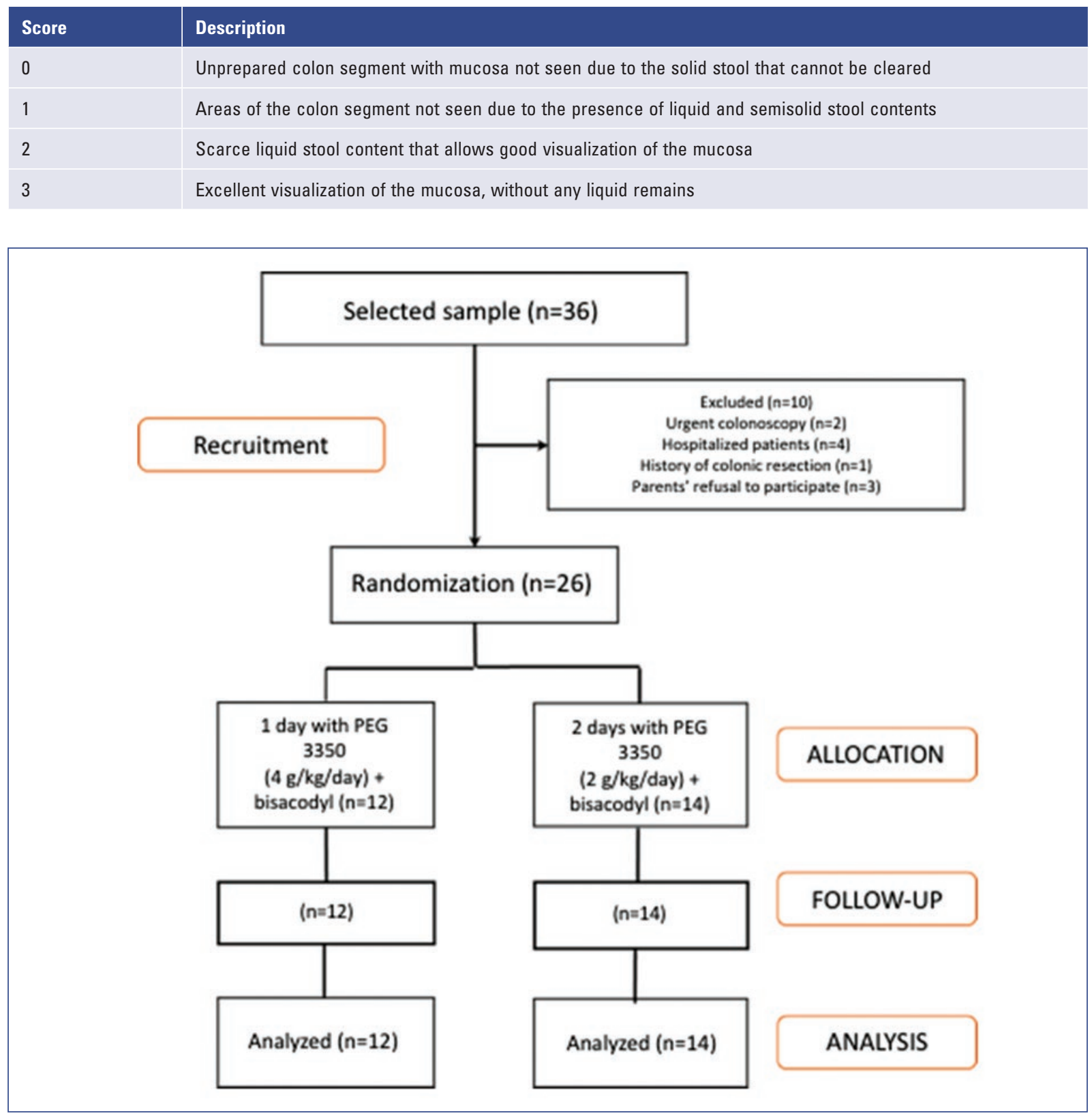

Figure 1. Diagram.

prospective study which determined that $1.9 \mathrm{~g} / \mathrm{kg}$ a day for two days along with a clear-liquids-diet yield clear stools in $>90 \%$ of patients $4,5,8,9$.

Currently, no standardized bowel preparation protocols in children exist. Therefore, an ample variety of methods exists, which vary significantly between medical centers and individual professionals. This lack of standardization makes it difficult to identify the efficacy and safety of the different regimes. Therefore, the objective of our study was to compare the tolerance, safety, and efficacy of two bowel preparations for colonoscopy. The first, one day of PEG 3350 (4 g/ $/ \mathrm{kg} /$ day) + bisacodyl 5-10 mg and the second, two days of preparation with PEG 3350 (2 g/kg/day) + bisacodyl 6-10 mg in pediatric patients attending the Hospital Infantil de México Federico Gómez (HIMFG).

\section{Methods}

A two-arm controlled randomized clinical trial with was conducted: 1 day of preparation with PEG 3350 (4 k/kg/day) + bisacodyl and two days of preparation 
with PEG 3350 (2 g/kg/day) + bisacodyl. From May to November 2016, children from 2 to 18 years of age attending the Department of Gastroenterology and the Thoracic Surgery and Endoscopy Service at the HIMFG were recruited for the study.

The Research and Ethics Committee previously approved the protocol with the registry number HIMAR 21/2016. To participate, parents signed an informed consent letter.

Patients programmed for ambulatory diagnostic and therapeutic colonoscopy were included. Patients with urgent colonoscopy, hospitalized, with a history of coIonic resection, with recent intestinal surgery (<28 days), known allergy to PEG or bisacodyl, chronic constipation causing fecal impaction and cardiac, hepatic or renal decompensated disease were excluded from the study. Patients in whom colonoscopy could not be performed due to lack of fasting on the day scheduled were eliminated.

\section{Randomization}

Patient allocation to each arm was randomized, the process of randomization was done before the start of the study, by using random numbers previously generated by a computer. To keep the allocation sequence hidden for the application of the intervention (1 day or 2 days), this was kept in closed and opaque envelopes until the patient was selected and the informed consent letter was signed. The person in charge of the intervention delivered the envelopes to the patients and assigned the intervention according to the corresponding card.

\section{Interventions}

Two interventions were performed: 1 day of preparation with PEG 3350 (4 g/kg/day) + bisacodyl and 2 days of preparation with PEG 3350 (2 g/ $/ \mathrm{kg} /$ day $)+$ bisacodyl. For the arm using a one-day preparation, the intervention consisted of the following:

- For patients weighing < $49.9 \mathrm{~kg}$ : PEG 3350 at a dose of $4 \mathrm{~g} / \mathrm{kg} /$ day mixed with a sports drink (Gatorade $®$ ) $240 \mathrm{ml}$ for every $17 \mathrm{~g}$ of PEG (maximum of 1.5 liters), administered in 4 hours the afternoon before the study + bisacodyl $5 \mathrm{mg}$ orally the night before the study.

- For patients weighing $\geq 50 \mathrm{~kg}:$ PEG 3350 at a dose of $4 \mathrm{~g} / \mathrm{kg} / \mathrm{day}$ (maximum of $238 \mathrm{~g}$ ) mixed 1.5 liters of a sports drink (Gatorade $\AA$ ), administered in 4 hours the afternoon before the study + bisacodyl $10 \mathrm{mg}$ orally the night before the study.
For those receiving two days of preparation with PEG 3350 (2 g/kg(day) + bisacodyl, the intervention consisted of the following:

- For patients weighing < $49.9 \mathrm{~kg}$; PEG 3350 at a dose $2 \mathrm{~g} / \mathrm{kg} /$ day mixed in a sports drink (Gatorade $®$ ) $240 \mathrm{ml}$ for every $17 \mathrm{gr}$ of PEG (maximum 1.5 liters), administered in 4 hours two afternoons before the study + bisacodyl $5 \mathrm{mg}$ orally daily, two nights before the study.

- For patients weighing $\geq 50 \mathrm{~kg}:$ PEG 3350 at a dose of $2 \mathrm{~g} / \mathrm{kg} /$ day (maximum of $238 \mathrm{~g}$ ) mixed with 1.5 liters of a sports drink (Gatorade $\AA$ ), administered in 4 hours two afternoons before the study + bisacodyl $10 \mathrm{mg}$ orally daily two nights before the study.

\section{Assessments of safety and tolerance of the interventions}

Tolerance assessments were measured using the percentage of solution the patient took during the days of preparation. The person in charge of the intervention calculated the quantity of liquid the patients should drink for one or two days according to the assigned intervention; a form to write the quantity of liquid drank by the patient was given. With these data, the percentage of liquids drank by the patient was calculated according to what was indicated and whether the patient required a nasogastric tube to complete treatment administration.

Safety assessments were measured through a questionnaire addressing the presence of nausea and vomit, as well as a physical examination to determine the hydration status and a glucose measurement using a reactive strip before the colonoscopy.

\section{Intervention's effectiveness assessments}

Effectiveness assessments were performed by the endoscopist using the Boston scale to evaluate the quality of cleanliness by segments: left, transverse, and right colon. Assignment to the procedure was blinded to the specialist performing the colonoscopy.

Two high-resolution colonoscopes were used: Fuji Film $₫$ EC-530 MP/LP Colonoscope (with an external diameter of $11 \mathrm{~mm}$ ) and Olympus $₫$ CF-Q150L// colonoscope (with an external diameter of $12.8 \mathrm{~mm}$ ). Colonoscopies were performed by physicians in training with the support of experienced endoscopists. Patients underwent general anesthesia applied by a pediatric anesthesiologist for all studies. 
Table 2. General patient data and comparison between the intervention groups

\begin{tabular}{|c|c|c|c|c|}
\hline & $\begin{array}{c}\text { All } \\
(n=26)\end{array}$ & $\begin{array}{l}\text { One day } \\
(n=12)\end{array}$ & $\begin{array}{l}\text { Two days } \\
(n=14)\end{array}$ & \multirow[t]{2}{*}{$\mathbf{p}$} \\
\hline & \multicolumn{3}{|c|}{ n (\%) } & \\
\hline Age (months) $)^{+}$ & $74(29-208)$ & $74(46-173)$ & $75.5(29-208)$ & 0.796 \\
\hline Female & $7(26.92)$ & $4(33.33)$ & $3(21.43)$ & 0.495 \\
\hline $\mathrm{Z}$ index of $\mathrm{BMI}^{+}$ & $0.45(-1.39$ to 2.15$)$ & $0.15(-1.39$ to 1.34$)$ & $0.75(-1.18$ to 2.15$)$ & 0.164 \\
\hline Constipation & $3(11.54)$ & $2(16.67)$ & $1(7.14)$ & 0.449 \\
\hline Indication: Lower Gastrointestinal Bleeding & $22(84.62)$ & $11(91.67)$ & $11(78.57)$ & 0.356 \\
\hline $\begin{array}{l}\text { Time between preparation and performance } \\
\text { of the study } y^{+}\end{array}$ & $15(14-18)$ & $15(14-16)$ & $16(14-18)$ & $\frac{0.127}{\frac{3}{5}}$ \\
\hline Histopathological diagnosis in colonoscopy & $18(69.23)$ & $10(83.33)$ & $8(57.14)$ & 0.149 \\
\hline $\begin{array}{l}\text { Compliance with the administration of PEG } \\
3350+\text { Bisacodyl }\end{array}$ & $26(100)$ & $12(100)$ & $14(100)$ & $\frac{-}{\circ}$ \\
\hline
\end{tabular}

+ Median (minimum-maximum); BMI, body mass index.

\section{Statistical analysis}

Measures of central tendency and dispersion according to the measuring scale of the variables. Quantitative variables were determined to have a non-normal distribution.

A Fisher's exact test and a Mann-Whitney $U$ test were calculated to compare if descriptive variables were similar between groups.

The proportion of tolerance, safety, extension of evaluation and quality of preparation between both groups was assessed. In tables with more than three variables, a $X^{2}$ test was used; for $2 \times 2$ tables, Fisher's exact test was applied. A Mann-Whitney $U$ test was used to compare scores of the Boston scale between both groups.

All analyses were done using the STATA software version 14.0.

\section{Results}

Between May and November 2016, 36 patients that required colonoscopy were identified at the HIMFG. A total of 10 patients were excluded from the study because they required urgent colonoscopy: four patients because they were hospitalized, one because of a previous history of colonic resection and three who did not agree to participate in the study (Figure 1).

Of the 26 patients that fulfilled the inclusion criteria, mean age was $93+54$ months (minimum of 2 years, maximum of 17 years), with a male predominance of
$73 \%$. Most patients had a history of lower gastrointestinal bleeding, which was the indication for the colonoscopy and in more than half of the cases pathological findings were found in the colonoscopy (Table 2).

Twenty-six patients were randomly assigned to two groups, according to the intervention. Group 1, which received a day of PEG 3350 (4 g/kg/day) + bisacodyl, included 12 patients; group 2, which received 2 days of PEG 3350 (2 g/kg/day) + bisacodyl, 14 patients. Groups were balanced since there was a less than $20 \%$ difference in the number of subjects included and analyzed (14\%) (Figure 1).

Comparisons included age, gender, $z$ index of the body mass index (BMI), history of constipation, lower gastrointestinal bleeding, time elapsed between preparation, the performance of the study and histopathological diagnosis for both groups, and no statistical difference was found between groups (Table 2). Regarding histopathological diagnosis, nine patients in the one-day group had a polyp, and one patient had colitis, while, in the two-day group, seven patients had a polyp, and one had a pseudopolyp; in the rest of the patients, the colonoscopy was normal $(n=8)$.

Treatment administration for bowel preparation (PEG $3350+$ bisacodyl) was administered in $100 \%$ of the patients, and none required placement of a nasogastric tube or hospitalization to complete the established dose.

Regarding the safety of the maneuvers, tolerance was analyzed considering the difficulty for administration by interrogating the parents, and no statistically significant 
Table 3. Comparison of tolerance and safety of the intervention between groups

\begin{tabular}{|c|c|c|c|c|}
\hline & & $\begin{array}{l}\text { One day } \\
(n=12)\end{array}$ & \multicolumn{2}{|l|}{$\begin{array}{l}\text { Two days } \\
(\mathrm{n}=14)\end{array}$} \\
\hline & & \multicolumn{3}{|c|}{ n (\%) } \\
\hline Tolerance & Difficulty for administration & $2(16.67)$ & $2(14.29)$ & $\stackrel{1}{2}$ \\
\hline Safety & Adverse effects & $4(33.33)$ & $2(14.29)$ & है \\
\hline & Hypoglycemia & $1(8.33)$ & $3(21.43)$ & $\therefore$ \\
\hline
\end{tabular}

* Without any significant differences between groups

difference was found between groups. Adverse events and hypoglycemia were documented to evaluate the safety. In the one-day bowel preparation group, four patients were reported to have adverse events that included headaches, abdominal pain, nausea, and vomit, meanwhile in the two-day bowel preparation group; two patients were reported to have adverse events that included nausea and headaches; there were no significant differences between groups. Before the colonoscopy, capillary glucose measurement was determined. It was found that in the one-day bowel preparation group, there was only one episode of hypoglycemia in the same patient that complained of a headache; in the two-day bowel preparation group, three cases of hypoglycemia were reported, without any accompanying symptoms, and without any significant statistical differences between groups (Table 3 ).

Regarding the extension of evaluation, full colonoscopy with visualization of the cecum and terminal ileum intubation was achieved in $83.3 \%$ of the patients that received the one-day regime, meanwhile, in the twoday group, such evaluation was achieved in $72.4 \%$ of the patients. Incomplete evaluations related to technical difficulties (not dependent on bowel cleanliness) or due to voluntary decisions to interrupt the colonoscopy advance in patients who underwent multiple polypectomies were performed, without any statistical differences between groups (Table 4).

When analyzing the efficacy of the maneuvers using the Boston scale, it was observed that in patients with one-day bowel preparation, the left colon showed a tendency for better grading when compared to patients with two-day bowel preparation, without any statistical significance. On the other hand, for the total score, which was obtained by adding the average scores for the right, left and transverse colon, there was a tendency for a better score in the patients with two-day bowel preparation, without a significant statistical difference.
Table 4. Comparison of the extension of evaluations between groups

\begin{tabular}{|l|c|c|}
\hline & $\begin{array}{c}\text { One day } \\
(\mathbf{n}=12)\end{array}$ & $\begin{array}{c}\text { Two days } \\
(\mathbf{n}=14)\end{array}$ \\
\cline { 2 - 3 } & \multicolumn{2}{|c|}{$\mathbf{n ( \% )}$} \\
\hline Transverse colon & $1(8.33)$ & $0(0)$ \\
\hline Ascending colon & $1(8.33)$ & $4(28.57)$ \\
\hline Cecum & $4(33.33)$ & $2(14.2)$ \\
\hline Ileum & $6(50)$ & $8(57.15)$ \\
\hline
\end{tabular}

* Without any significant differences between groups

In the transverse and right colon, there were no statistical differences in the scores (Table 5).

Another way of evaluating efficacy through the Boston scale is to identify the proportion of subjects with "good" or "excellent" score with the bowel preparation (excellent $=3$ and good=2 in each segment of the colon). According to this scale, it was observed that the one-day bowel preparation group had a greater proportion of patients with "excellent" and "good" scores compared with those in the two-day bowel preparation group for the left colon, with a statistical significance (100\% vs. $71.43 \%, p=0.044$ ) (Table 5). Analysis of the final score for both groups showed that the one-day bowel preparation group had "excellent" or "good" scores in $83.33 \%$ of the patients and the two-day bowel preparation group had "excellent" or "good" scores in $57.14 \%$ of the cases $(p=0.14)$.

\section{Discussion}

This study found that bowel preparation for colonoscopy using one day of PEG 3350 (4 g/ $/ \mathrm{kg} /$ day $)+$ bisacodyl was partially more effective compared to two 
Table 5. Comparison of the efficacy of the intervention between the two groups using the Boston scale

\begin{tabular}{|c|c|c|}
\hline \multirow[t]{2}{*}{ Score } & $\begin{array}{l}\text { One day } \\
(n=12)\end{array}$ & $\begin{array}{c}\text { Two days } \\
(\mathrm{n}=14)\end{array}$ \\
\hline & \multicolumn{2}{|c|}{ Median (min-max) } \\
\hline $\begin{array}{l}\text { Left colon } \\
\text { Transverse colon } \\
\text { Right colons } \\
\text { Total }\end{array}$ & $\begin{array}{l}2(2-3) \\
3(2-3) \\
3(0-3) \\
7(6-9)\end{array}$ & $\begin{array}{c}2(1-3) \\
2.5(1-3) \\
2.5(1-3) \\
7(5-9)\end{array}$ \\
\hline & \multicolumn{2}{|c|}{ n (\%) } \\
\hline $\begin{array}{l}\text { Left colon } \\
\text { Excellent/Good } \\
\text { Bad/Inadequate }\end{array}$ & $\begin{array}{c}12(100)^{*} \\
0(0)\end{array}$ & $\begin{array}{c}10(71.43)^{*} \\
4(28.57)\end{array}$ \\
\hline $\begin{array}{l}\text { Transverse colon } \\
\text { Excellent/Good } \\
\text { Bad/Inadequate }\end{array}$ & $\begin{array}{c}12(100) \\
0(0)\end{array}$ & $\begin{array}{c}13(92.86) \\
1(7.14)\end{array}$ \\
\hline $\begin{array}{l}\text { Right colon } \\
\text { Excellent/Good } \\
\text { Bad/Inadequate }\end{array}$ & $\begin{array}{c}10(83.33) \\
2(16.67)\end{array}$ & $\begin{array}{c}13(92.86) \\
1(7.14)\end{array}$ \\
\hline $\begin{array}{l}\text { Total } \\
\text { Excellent/Good } \\
\text { Bad/Inadequate }\end{array}$ & $\begin{array}{c}10(83.33) \\
2(16.67)\end{array}$ & $\begin{array}{l}8(57.14) \\
6(42.86)\end{array}$ \\
\hline
\end{tabular}

days of PEG 3350 (2 g/kg/day) + bisacodyl. Overall, both bowel preparation regimes were well tolerated and had similar adverse effects, without statistically significant differences between groups.

Tolerance to medications used for colonoscopy preparation was measured using completeness of administration. In this regard, Najafi et al. ${ }^{8}$ reported global completeness of $92 \%$, as well as Abbas et al. ${ }^{10}$, who reported that $93.5 \%$ of children completed at least $75 \%$ of the preparation within a mean time of $120-219 \mathrm{mi}-$ nutes. The research group led by Phatak ${ }^{11}$ reported completeness of administration in $95 \%$ of patients. In our patients, only $15.38 \%$ reported bad tolerance, but despite that, completeness was $100 \%$; these high levels of completeness may be the result of several factors, such as that PEG does not significantly modify the taste of beverages. In both groups, the volume of liquid was proportional to the calculated quantity of PEG using the weight of the patient, having 1.5 liters as a limit: preparation was divided into eight doses every 30 minutes to be completed within four hours, which improved the acceptance of said volume of liquid.

Adverse effects reported by Phatak et al. ${ }^{11}$ included mild nausea (19\%), abdominal pain (11\%) and vomit
(4\%). Najafi et al. ${ }^{8}$ reported the presence of abdominal pain and nausea, without a statistically significant difference between both groups. Abbas et al. ${ }^{10}$ reported nausea or vomit $(60 \%)$, abdominal pain or colic $(44 \%)$ and fatigue or weakness (40\%). The adverse effects identified in our study were similar to those described by these authors and included a headache, abdominal pain, nausea and vomit, all referred as mild. Adverse effects presented in $34.15 \%$ of the cases $(n=9)$, without a difference between groups. No patient suffered dehydration, and in $15.38 \%(n=4)$, the presence of hypoglycemia was reported, without a statistically significant difference. These results should be taken with caution, since, despite no statistically significant difference was demonstrated, this may be due to the small sample size.

Efficacy was evaluated through the Boston scale. In this regard, Phatak et al. ${ }^{11}$ used PEG (2 g/kg/day) + bisacodyl for two days, achieving a quality of cleanliness reported as "excellent" or "good" in the right and left colon in $92 \%$ and $93 \%$, respectively. In comparison, our results were similar for the right colon: the two-day bowel preparation group had an "excellent" or "good" score in $92.86 \%$. In contrast, in the one-day group, it was observed that $100 \%$ of the subjects had "excellent" or "good" scores. For the left colon, the two-day group achieved "good" or "excellent" cleanliness in $71.43 \%$, a lower percentage of that reported by Phatak et al., whereas $100 \%$ of the patients achieved a "good" or "excellent" quality of cleanliness in the one-day group"

Najafi et al. ${ }^{8}$ evaluated two colon preparations and obtained an "excellent" or "good" efficacy in the oneday group in $70 \%$ of the cases and $72 \%$ in the two-day group. When comparing these results with ours, efficacy in the two-day group was similar. However, the quality of preparation was better in the one-day group (72\% vs. $100 \%)$.

Abbas et al..$^{10}$ used PEG for colon preparation and reported a total mean score of 6.16 on the Boston scale. In contrast, we observed a greater efficacy in both groups (global Boston scale in the one-day group of 7.41 vs. 6.7 in the two-day group). This result was possibly influenced by the additional effect of bisacodyl and that PEG 3350 was administered in a greater dose ( $4 \mathrm{~g} / \mathrm{kg} / \mathrm{day})$ in the one-day group. Conversely, Sahn et al. ${ }^{12}$ administered a one-day colonoscopy bowel preparation with PEG 3350 (4 g/kg/day) + bisacodylin 155 pediatric patients. Our results were similar since "excellent" or "good" cleanliness was reported in $77 \%$ of the cases; meanwhile, in our study, these were found in $83.3 \%$ of the subjects. Nevertheless, these results 
should be taken with caution since evaluations were incomplete in some patients, regarding the extension of the colonoscopic study.

Concerning the potential confounding variables, both groups were balanced; patients with constipation represented $16.67 \%$ and $7.14 \%$ in the one-day and two-day groups, respectively. Moreover, the time from completing preparation and performance of the study was $15.04 \pm 0.83$ hours in the one-day group vs. $15.47 \pm$ 1.2 hours in the two-day group, without any statistically significant differences.

Regarding the extension of the evaluation, Abbas et al. ${ }^{10}$ reported that every colonoscopy was completed up to the cecum and in 844 of the cases visualization of the terminal ileum was possible. Phatak et al. ${ }^{11} \mathrm{ob}$ served that in $70 \%$ of the children evaluation of the terminal ileum was achieved. Our results differ since the cecum could only be reached in $83 \%$ of the cases and the terminal ileum in $50 \%$ of the patients in the one-day group. The cecum was reached in $71 \%$ of the cases, and the terminal ileum was evaluated in $57 \%$ in the two-day group, which represents a lower proportion compared to most of the previous studies, most of these due to technical issues and none due to lack of cleanliness in the area.

Our study had several limitations. One of the main limitations was the small sample size. Thus, results should be interpreted as trends and not as conclusions. On the other hand, the intervention could not be blinded to participants due to the way it was indicated, tolerance assessment was subjective, and the safety assessments were insufficient. Ideally, measurement of serum electrolytes and creatinine should have been performed before and after bowel preparation. The statistical power of the results in the Boston scale was calculated for the total score, where the power of $22 \%$ was reported. With the obtained results, the sample size was recalculated to detect statistical differences in the total score of the Boston scale, and it was determined that at least 94 patients per group be required. Nevertheless, when calculating the sample size using the scores of the left colons using the Boston scale, this yielded a total of 35 patients per group. Another limitation of our study was the low rate of visualization of the cecum and intubation of the terminal ileum.

Considering these factors, we recommend a larger sample size or a multicentric study to demonstrate the superiority of bowel preparation for colonoscopy using one or two days of PEG 3350 + bisacodyl, regarding efficacy and safety.

In conclusion, we found no differences regarding tolerance and safety between bowel preparation for colonoscopy using one day of PEG 3350 (4 g/ $/ \mathrm{kg} /$ day $)+$ bisacodyl and a two-day preparation with PEG 3350 $(2 \mathrm{~g} / \mathrm{kg} /$ day $)+$ bisacodyl in a pediatric population.

\section{Ethical disclosures}

Protection of human and animal subjects. The authors declare that no experiments were performed on humans or animals for this study.

Confidentiality of data. The authors declare that they have followed the protocols of their work center on the publication of patient data.

Right to privacy and informed consent. The authors declare that no patient data appear in this article.

\section{Conflicts of interest}

The authors declare no conflicts of interest.

\section{References}

1. Aguirre L. Colonoscopía Diagnóstica en Pediatría. En: Córdova De la Torre, editors. Procedimientos endoscópicos en gastroenterología. Mexico City: Médica Panamericana; 2009. pp. 613-8.

2. Calderwood A, Thompson K. Good is better than excellent: Bowel preparation quality and adenoma detection rates. Gastrointest Endosc. 2015;81:691-9.

3. Lai E, Calderwood A, Doros G, Fix O, Jacobson B. The Boston bowel preparation scale: A valid and reliable instrument for colonoscopy-oriented research. Gastrointest Endosc 2009;69:620-5.

4. A consensus document on bowel preparation before colonoscopy: Prepared by a Task Force from the American Society of Colon and Rectal Surgeons (ASCRS), the American Society for Gastrointestinal Endoscopy (ASGE), and the Society of American Gastrointestinal and Endoscopic Surgeons (SAGES). Gastrointest Endosc. 2006:63:894-9.

5. Hunter A, Mamula P. Bowel preparation for pediatric colonoscopy procedures. J Pediatr Gastroenterol Nutr. 2010;51:254-61.

6. Alper A, Pashankar D. Polyethylene glycol: A game-changer laxative for children. J Pediatr Gastroenterol Nutr. 2013:57:134-40.

7. Parra-Blanco A, Ruiz A, Álvarez-Lobos M, Amorós A, Gana JC, lbáñez P, et al. Achieving the best bowel preparation for colonoscopy. World $J$ Gastroenterol. 2014:20:17709-26.

8. Najafi M, Fallahi G, Motamed F, Farahmand F, Khodadad A, GhajarzadehW M, et al. Comparison of one and two-day bowel preparation with polyethylene glycol in pediatric colonoscopy. Turk J Gastroenterol. 2015;26:232-5

9. Walia R, Steffen R, Feinberg L, Worley S, Mahajan L. Tolerability, safety, and efficacy of PEG 3350 as a 1-day bowel preparation in children. J Pediatr Gastroenterol Nutr. 2013;56:225-8.

10. Abbas M, Nylund C, Bruch C, Nazareno L, Rogers P. Prospective evaluation of 1-day polyethylene glycol-3350 bowel preparation regimen in children. J Pediatr Gastroenterol Nutr. 2013;56:220-4.

11. Phatak U, Johnson S, Husain S, Pashankar D. Two-day bowel preparation with polyethylene glycol 3350 and bisacodyl: a new, safe, and effective regimen for colonoscopy in children. J Pediatr Gastroenterol Nutr. 2011;53:71-4.

12. Sahn B, Chen-Lim M, Ciavardone D, Farace L, Jannelli F, Nieberle M, et al. Safety of a 1-day polyethylene glycol 3350 bowel preparation for colonoscopy in children. J Pediatr Gastroenterol Nutr. 2016;63:19-24. 